\begin{tabular}{|c|c|c|c|c|}
\hline 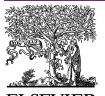 & Opinion & TRENDS in Cognitive Sciences & Vol.8 No.3 March 2004 & $\begin{array}{l}\text { Full text provided by www.sciencedirect.com } \\
\text { science doirect. }\end{array}$ \\
\hline
\end{tabular}

\title{
Can language restructure cognition? The case for space
}

\author{
Asifa Majid ${ }^{1}$, Melissa Bowerman ${ }^{1}$, Sotaro Kita ${ }^{2}$, Daniel B.M. Haun ${ }^{1,3}$ \\ and Stephen C. Levinson ${ }^{1}$
}

${ }^{1}$ Max Planck Institute for Psycholinguistics, Postbus 310, 6500 AH Nijmegen, The Netherlands
${ }^{2}$ University of Bristol, Dept of Experimental Psychology, 8 Woodland Road, Bristol BS8 1TN, UK
${ }^{3}$ Institute of Cognitive Neuroscience, University College London, Alexandra House, 17 Queen Square, London WC1N 3AR, UK

Frames of reference are coordinate systems used to compute and specify the location of objects with respect to other objects. These have long been thought of as innate concepts, built into our neurocognition. However, recent work shows that the use of such frames in language, cognition and gesture varies crossculturally, and that children can acquire different systems with comparable ease. We argue that language can play a significant role in structuring, or restructuring, a domain as fundamental as spatial cognition. This suggests we need to rethink the relation between the neurocognitive underpinnings of spatial cognition and the concepts we use in everyday thinking, and, more generally, to work out how to account for cross-cultural cognitive diversity in core cognitive domains.

Think where you left your glasses. Of course, they were to the right of the telephone! This is the sort of everyday coding of spatial location we use. But some people in other cultures think differently about the same situation: they would code the glasses as being on the telephone's own left side, or even as being north-east of the phone! Understanding these differences and their source is what this article is about. As the scene is the same, the differences in coding are clearly something we bring to the scene - what Gestalt theorists called a 'frame of reference', or a coordinate system, which we impose on the objects to get a specified direction for the glasses with respect to the telephone.

There has been a great deal of thought about spatial frames of reference (FoR) in psychology, neurocognition, linguistics and elsewhere. Most of this literature privileges egocentric coordinates, as exemplified in 'the glasses are to the right of the telephone': Kant argued elegantly that the human body frame is the source of our basic intuitions about the nature of space [1], a thought echoed by many modern psychologists [2]. The emphasis in modern psychology on the primacy of anthropomorphic and relativistic space concepts [3,4] neglects other work in psychology and neurocognition [5] and the facts of cultural and linguistic diversity, the focus of this article. This broader perspective recognizes not only egocentric coordinate systems, but also two distinct types of allocentric ones: those based on object-centred coordinates, and those based on absolute coordinates like north/south/ east/west. At a level of abstraction we can talk of just three FoRs, which we will call the Relative (roughly, egocentric), the Intrinsic (object-centred) and the Absolute (using fixed bearings like north).

In this article we show that many languages make little or no use of the Relative FoR, instead emphasizing one or more of the other frames, and, more surprising perhaps, that speakers of these languages appear to code their everyday non-linguistic spatial representations in line with their linguistic FoRs, neither speaking nor thinking in terms of the glasses being to the right of the phone. This raises a host of puzzles: How could such cognitive diversity arise? Could language difference be the root - is this a 'Whorfian' effect of language on cognition? Or are (noncultural) environmental factors at work? What about child development - are some FoRs easier to acquire than others?

\section{Frames of reference and linguistic diversity}

We start with a review of recent findings about linguistic diversity, concentrating on spatial descriptions on the horizontal plane. Recent research has shown that languages differ in the availability of FoRs for describing the relationship between small-scale, manipulable objects in 'table-top space' - that is, non-geographic space. Figure 1 illustrates the three FoRs, Relative, Absolute and Intrinsic, which can be thought of as different strategies for specifying the spatial relationship between the thing to be located (referent or figure) and the landmark (or ground object).

Languages have specialized expressions for one, two or all three FoRs [6-21]. The frequency and range of application of these FoRs differ across languages (see Box 1, Table I). English speakers use two different FoRs to describe spatial relationships in table-top space: they say either 'the fork is to the left of the spoon' (Relative FoR) or 'the fork is beside the spoon' (Intrinsic FoR). They do not say 'the fork is to the north of the spoon'; they restrict their use of the Absolute FoR to large-scale, geographical descriptions. However, speakers of Guugu Yimithirr (Australia) use only the last kind of description; they do not have available either a Relative or an Intrinsic FoR. The Absolute FoR is used even to describe the location of 


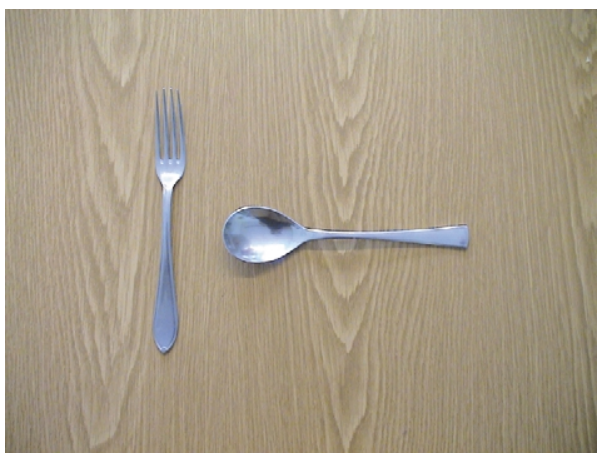

Relative: The fork is to the left of the spoon

Absolute: The fork is to the north of the spoon

Intrinsic: The fork is at the nose of the spoon

$\overline{\text { TRENDS in Cognitive Sciences }}$

Figure 1. Descriptions in Relative, Absolute and Intrinsic frames of reference. The spatial relationship between objects in table-top space, like the fork and the spoon, can be described in different ways. In the Relative FoR (dominant in English, Dutch and Japanese), the viewer's perspective is used, giving rise to descriptions such as 'the fork is to the left of the spoon'. Notice that if you go around to the other side of the table, the fork is now properly described as 'to the right of the spoon'. In an Absolute FoR an external framework is applied instead. This can be composed of cardinal directions such as north-south-east-west, used by speakers of Arrernte (Australia), or an uphill-downhill axis like the one used by speakers of Tzeltal (Mexico). So, as a speaker of Arrernte you would say 'the fork is to the north of the spoon', and as a Tzeltal speaker 'the fork is uphill of the spoon'; unlike in the Relative FoR, the same description is applied to the scene from whichever side it is viewed. Finally, in an Intrinsic FoR, you could describe the spatial relations between the fork and spoon without reference to either yourself or any other external system of coordinates. For instance, you could say 'the fork is at the nose of the spoon', as speakers of Mopan (Belize) or Totonac (Mexico) do.

an object on a body part - a Guugu Yimithirr speaker would say 'There's an ant on your south leg' [9].

Frames of reference and their underlying computations To use linguistic terms specialized for different FoRs, distinct cognitive computations are required. In an Intrinsic FoR, the cognitive system must 'parse' objects into their major parts (such as 'front', 'back' and 'side') to find the named facets of the landmark object used in intrinsic descriptions; for example, 'by the side of the bed', 'at the front of the truck'. The criteria for doing this vary across languages. Tzeltal (Mexico) has a strictly geometric method, based on the volumetric properties of the landmark object regardless of its orientation [22], but English uses mixed strategies based on (a) an oriented template giving 'top', 'bottom' and 'sides', and (b) functional criteria, so the 'front' of a building is the side you enter, the 'front' of a TV the side one faces to watch it, etc. [13]. Once the object has been parsed, a second step is required: to project into space from a designated facet of the object, so that we can say, for example, 'the statue is in front of the cathedral' even though it is 200 metres away [23].

There is a tight connection between the Relative FoR and the Intrinsic FoR: it seems that you cannot have a Relative FoR without an Intrinsic FoR $[13,17]$. Like the Intrinsic FoR, the Relative FoR requires 'parsing' of objects - most importantly, a parsing of the self into front, back, left and right. This parsing is then projected into space, so that objects can be to my left and right side even when I am not in contact with them. However, if I say 'the fork is on the left' or 'the fork is in front of me', meaning in the space adjacent to my left side or my front side, this is not the Relative FoR, but still the Intrinsic FoR. This is because only a binary relation is being coded: the relationship between myself and the fork. It is a Relative FoR when a ternary relationship is encoded; for example, 'the fork is to the left of the spoon' or 'the fork is in front of the spoon': now the relationship between the fork and the spoon is encoded from the perspective of a third participant, the viewer. Some sentences in English are ambiguous between these two FoRs.

The Relative FoR can be 'lifted' from the self, rotated and applied to other people and objects [24]. So, if Bill and I are sitting on opposite sides of the table in Figure 1, then from my perspective I can say that 'the fork is to the left of the spoon' but I can also ask Bill to 'pass me the fork that is to the right of the spoon', i.e. using his perspective.

The computations required if you use an Absolute FoR in all situations, from table-top space to geographic, are of a quite different kind. For example, you must know at all times and in all locations where your conventional fixed bearings are (thus in Guugu Yimithirr local 'north' is centred on approximately $\mathrm{N} 017^{\circ}$, in Tzeltal 'uphill' is focussed on approximately $\mathrm{N} 345^{\circ}$ ). To do this, you must run a 'mental compass', a constant background computation of direction. You must code all percepts that you might later want to talk about in terms of such fixed bearings, so you can say (or for that matter, think) 'I must have left my glasses to the north of the telephone'. You need to maintain mental maps correctly oriented so that you can calculate the bearing between any two points you might want to talk about. You also need to dead-reckon your current location so that you can correctly describe where unseen points are from the current location.

\section{Frames of reference and cognition}

Do the computations needed for speaking languages with different FoRs affect only the processes of speaking and comprehending? Or do they have deeper cognitive consequences as well? Some say the answer is no. According to one view, spatial cognition is inherently 'dynamic, egocentric and primitive', such that everyone, regardless of language, uses an egocentric representation to solve nonlinguistic spatial tasks [4]. Another view is that everyday spatial cognition requires people to maintain multiple FoRs, and that languages merely recruit from these antecedent systems $[25,26]$. We can decide between these various alternatives by drawing on an interesting contrast between Absolute and Relative FoRs: under rotation, the Absolute FoR stays stable whereas the Relative FoR changes. That is, if I turn $180^{\circ}$, the space to the north of me remains the same but the space to the left of me rotates. How do speakers of languages with different FoRs behave on non-linguistic tasks under rotation?

A set of languages were classified as Absolute, Relative or mixed according to the FoR their speakers preferred to use when describing the relationship between objects in table-top space [16]. In subsequent non-linguistic tasks, speakers of these languages were shown a spatial relation between objects on a table, or a spatial trajectory, and then 


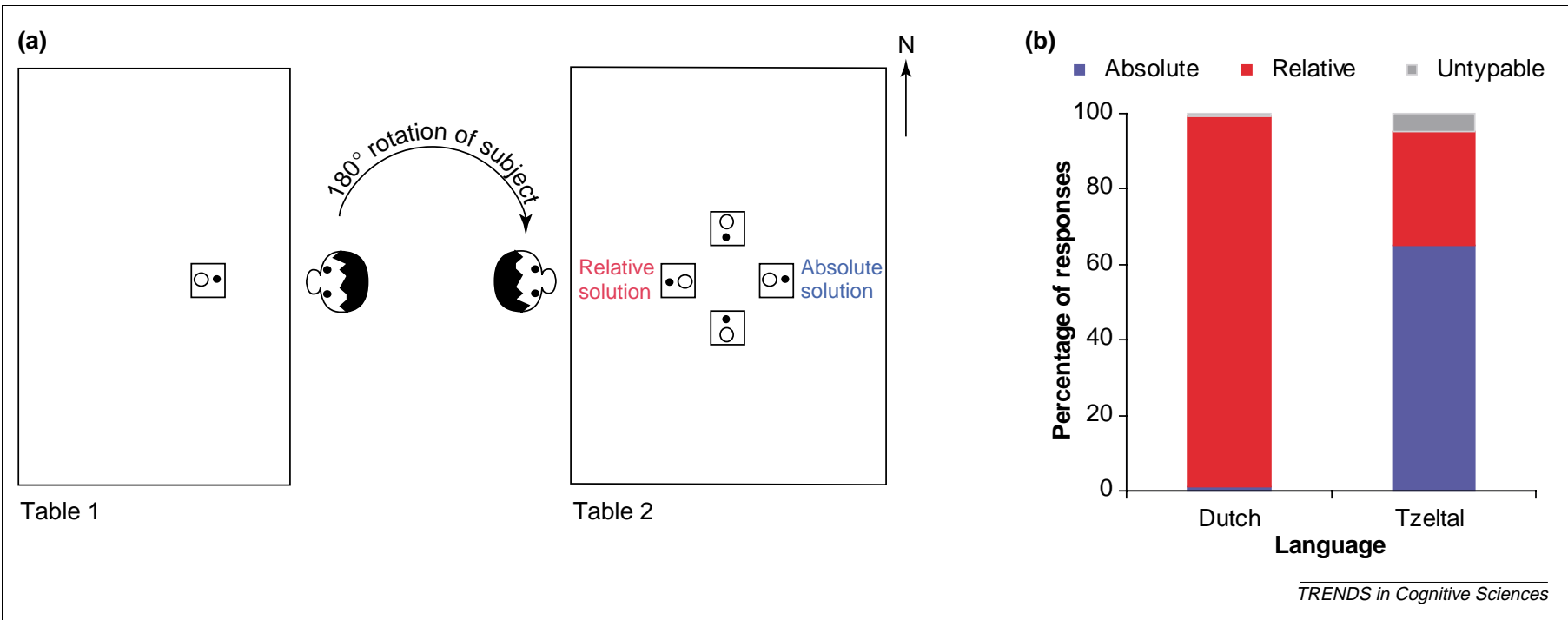

Figure 2. Memory for spatial configuration: 'the chips task'. (a) Participants saw on Table 1 a card printed with a large and a small dot arranged with the small dot towards them, away from them, to the left, or to the right. After a 30 -s delay, they were rotated through $180^{\circ}$ and led to Table 2 , where they were asked (in the local language) to identify from a set of four cards the similar or counterpart card to the one they had seen before. Each participant had eight trials, which varied in the arrangement of the dots. Responses that preserved sameness in egocentric coordinates were coded Relative, those that preserved Absolute coordinates were coded Absolute, and other responses were coded 'Untypable'. (b) The results for just two languages, Dutch and Tzeltal. The Relative/Absolute trend matches the preferred linguistic FoR: Dutch responses were overwhelmingly Relative whereas Tzeltal responses were overwhelmingly Absolute. The proportionately larger Tzeltal inconsistency can be attributed to the fact that the data are from an unschooled peasant population. However, if we look for 'consistent coders', that is, coders who give the same response on 6 out of 8 trials, then over $80 \%$ of Tzeltal speakers are 'Absolute thinkers' (Adapted from [13], pp. 159-160, by permission of Cambridge University Press).

rotated $180^{\circ}$ to face another table, where they had to solve a spatial task. Some of the tasks involved memory for the spatial configuration of objects (see Figure 2), others involved memory for motion and path-direction (Figure 3), and still others tapped spatial reasoning (Figure 4) $[13,16,20,27]$. These experiments have shown that people do indeed use different non-linguistic FoRs to do the same tasks, and that these non-linguistic FoRs align with the preferred FoR of their language.
It has been suggested that these differences are the result of a confound - for example, that testing conditions were not sufficiently controlled across populations [28]. But when environmental features were controlled, the same correlation between linguistic and non-linguistic representations was obtained [27]. Alternative explanations invoking an environmental or cultural confound [29] also seem unviable. Absolute behaviour on non-linguistic tasks has been demonstrated with speakers of the (a)

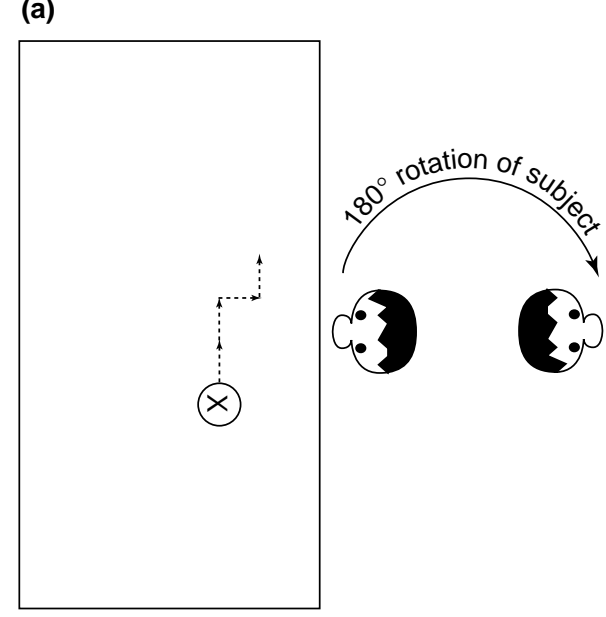

Table 1

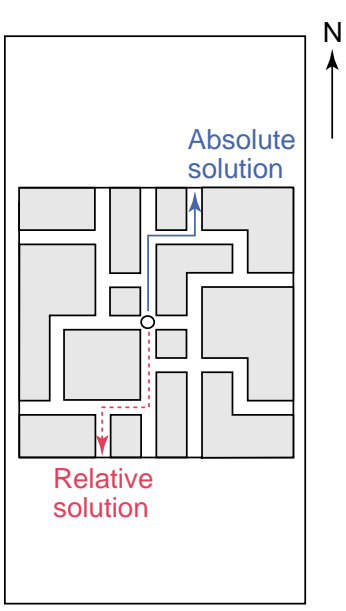

Table 2

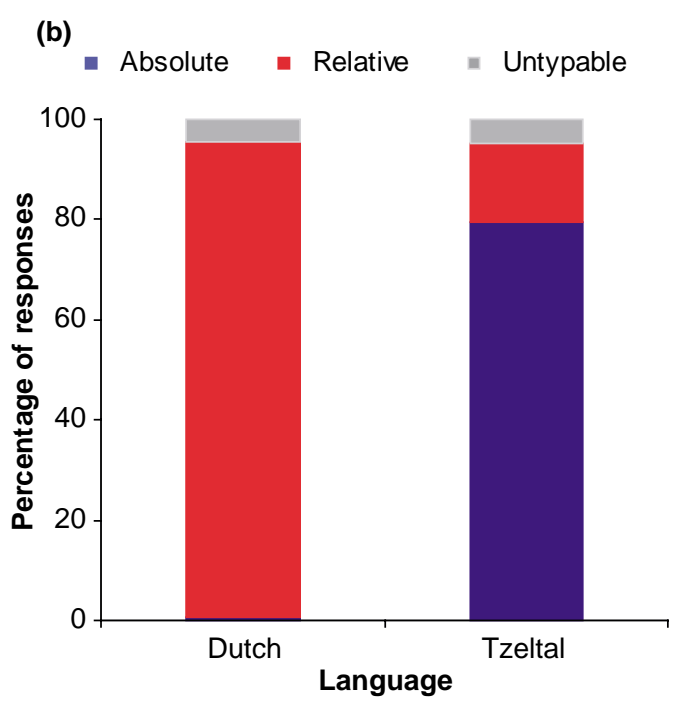

TRENDS in Cognitive Sciences

Figure 3. Memory for motion and path-direction: 'Eric's maze'. (a) The experimenter moved a toy man along a path on Table 1. After a delay, the participant was rotated through $180^{\circ}$ and led to Table 2, where there was a maze. The maze had several possible paths, and the participant was asked to choose the path that the toy man had followed. Each participant had five trials. For each target path the toy man had travelled along on Table 1, there were two corresponding paths on the maze on Table 2: one that preserved Relative coordinates (shown in red), and one that preserved Absolute coordinates (shown in blue). (b) The results for Dutch and Tzeltal. As in Figure 2, we see that the Relative/Absolute trend matches the preferred linguistic FoR: Dutch participants gave Relative responses whereas Tzeltal participants gave predominantly Absolute responses (Adapted from [13], pp. 160-162, by permission of Cambridge University Press). 


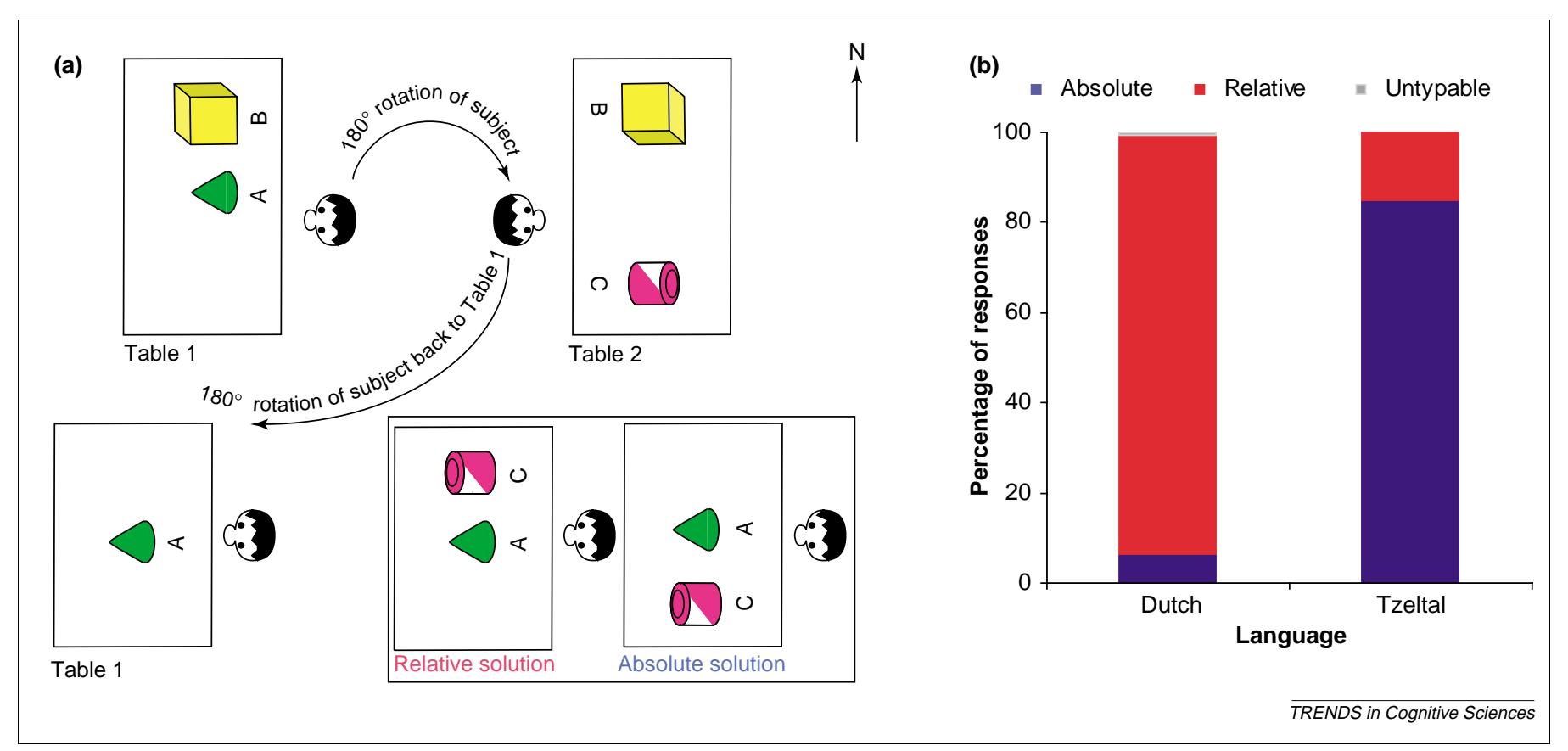

Figure 4. Spatial reasoning: making a transitive inference. (a) Transitive inferences (e.g. if $A$ is bigger than $B$, and $B$ is bigger than $C$, then $A$ is bigger than $C$ ) can be drawn on the basis of non-linguistic arrays. In this experiment, participants saw a cube and a cone arranged in a particular configuration on Table 1 , and were then rotated through $180^{\circ}$ to Table 2 where they saw a cube and a cylinder. Finally they were rotated back to Table 1 , where the cone was standing alone. Their task was to place the cylinder next to the cone, keeping the location consistent with what they had seen before. There were five trials using the transverse axis. There are two ways to perform this task: one using Relative coding (the cube is to the right of the cone, and the cylinder is to the right of the cube, therefore the cylinder is to the right of the cone), and the other using Absolute coding (the cube is to the south of the cone, and the cylinder is further south of the cube, therefore the cylinder is to the south of the cone). (b) Once again the results for Dutch and Tzeltal show that the Relative/Absolute trend matches the preferred linguistic FoR: Dutch participants gave Relative responses whereas Tzeltal participants gave Absolute responses (Adapted from [13], pp. 162-167, by permission of Cambridge University Press).

Absolute languages Guugu Yimithirr (Australia), Arrernte (Australia), Hai//om (Namibia), Tzeltal (Mexico), Longgu (Solomons), Belhare (Nepal) and Balinese (Indonesia) $[13,16,20]$, whereas Relative behaviour has been shown with speakers of the Relative languages Dutch, Japanese and Yukatek (Mexico) $[13,16]$. The variation in cultural and environmental factors within groups is as large as the variation between groups: Absolute and Relative languages are spoken in a variety of different ecological environments, with people of different subsistence modes (see Box 1).

In this sample of 10 communities there does appear to be one association involving an ecological factor: living in an urban environment is associated with using a Relative FoR and living in a rural environment with using an Absolute FoR. Could the urban-rural divide explain nonlinguistic cognition on the rotation tasks? This seems unlikely for two reasons. First, there are examples of rural communities that predominantly use a Relative FoR in both language and non-linguistic cognition (e.g. the Yukatek). Second, it is unclear what mechanism would make urban-dwellers Relative and rural-dwellers Absolute in their non-linguistic cognition. One possibility could be literacy: increased literacy, which correlates with urbanization, could encourage a Relative FoR. But within populations there is no correlation between literacy and preferred FoR on cognitive tasks (with the single exception of Belhare) [13]. Although there is no attested mechanism that explains why urban and rural communities would differ in their FoR, there are attested cognitive mechanisms that can explain how language can affect cognition (see Box 2).

\section{Frames of reference and gesture}

The alignment of linguistic and non-linguistic FoRs is pervasive beyond the experimental contexts mentioned above, as shown by evidence from spontaneous co-speech gestures. Iconic gestures (e.g. depicting a movement trajectory) and pointing gestures can reflect aspects of a speaker's non-linguistic spatial representation [30]. Analysis of gestures in different cultures reveals that the default gestural FoR matches the predominant linguistic FoR.

Thus, speakers of Absolute languages such as Guugu Yimithirr and Tzeltal typically encode directionality in the Absolute FoR $[9,10,13]$. For example, an object moving west is represented with a hand movement towards the west (to the left if facing north, and straight away from the body if facing west). By contrast, speakers of Relative languages such as English, Japanese and Turkish typically encode a movement from left to right in their viewing field with a hand movement to the right, regardless of which direction they are facing at the time of speech [31]. Speakers of Intrinsic languages like Mopan (Belize) typically encode directionality in the Intrinsic FoR; for example, change of location resulting from human locomotion is almost exclusively represented from the perspective of the mover, as a hand movement sagittally away from the speaker [32]. The default gestural FoR is maintained at all times: for example, English, Turkish and Japanese speakers code Relative left and right directions in their gestures even when they don't refer to the FoR in their accompanying speech [31].

\section{Frames of reference and child development}

The research discussed above shows that adult speakers of some languages privilege the Absolute FoR in both 


\section{Box 1. Frames of reference and ecological determinism}

The correlation between the linguistic FoR people use and their performance on non-linguistic tasks has been interpreted as a Whorfian effect, that is, as an effect of linguistic categories on non-linguistic ones $[13,16,27]$. But perhaps the correlation can be explained by some third intervening variable. Three types of intervening variables have been proposed.

The first is that environment $[28,40]$ shapes both linguistic and nonlinguistic categories. Salient differences in environment such as urban vs. rural, or open terrain vs. dense forest, could affect both language and cognition. For example, one hypothesis is that rural or small-scale societies lack cardinal directions, whereas urban societies are more mobile and so tend to use Absolute systems [41]. A contradictory hypothesis is that the more 'insular' or geographically cohesive a group is (i.e. rural communities), the more likely it is to have an Absolute FoR [28].

A second possibility is that action $[25,26]$ is the intervening variable: different actions might call for different FoRs, so differences in habitual action, perhaps reflected in subsistence patterns, could give rise to differential use of FoRs in both language and cognition.

A third possibility is that global cognitive styles like individualism versus collectivism [42] mediate between language and cognition. Perhaps speakers of Relative languages are more individualist and so make use of an egocentric FoR, whereas speakers of Absolute languages are collectivist and so use a FoR that is shared by the group.

Table I shows 20 languages with their associated FoRs, along with information about environment (operationalized as the ecological zone speakers of that language inhabit), dwelling (predominantly rural or urban), and subsistence patterns (representing habitual action). Individualism vs. collectivism was assigned using the definitions given by Greenfield et al. [42]; all the language groups were judged to be collectivist with the exception of Dutch and English. None of the factors appears to determine the FoR, although there might be an association between urban-dwelling and use of a Relative FoR. Of course, the lack of a relationship does not prove that environment, action or individualism vs. collectivism have no impact on choice of FoR, but it does suggest that there is no simple determinism between ecological factors, or global cognitive style, and whether a speaker will use Intrinsic, Relative or Absolute FoR to describe relationships between objects in table-top space.

Table I. Frames of reference and ecological determinism

\begin{tabular}{|c|c|c|c|c|c|c|c|c|}
\hline \multirow[t]{2}{*}{ Language } & \multirow[t]{2}{*}{ Country } & \multirow[t]{2}{*}{ Family } & \multicolumn{3}{|c|}{ Linguistic frame of reference } & \multirow{2}{*}{$\begin{array}{l}\text { Ecological zone } \\
\text { or zones }\end{array}$} & \multirow[t]{2}{*}{ Dwelling } & \multirow{2}{*}{$\begin{array}{l}\text { Subsistence } \\
\text { mode }\end{array}$} \\
\hline & & & Intrinsic & Relative & Absolute & & & \\
\hline Arrernte & Australia & Pama Nyungan & $x$ & & $\mathbf{x}$ & $\mathrm{D}$ & $\mathrm{R}$ & $\mathrm{H}-\mathrm{G}$ \\
\hline Balinese & Indonesia & Austronesian & $x$ & $x$ & $\mathbf{x}$ & $\mathrm{T}$ & $\mathrm{R}$ & StA \\
\hline Belhare & Nepal & Tibeto-Burman & $x$ & $x$ & $\mathbf{x}$ & H SubT, A & $\mathrm{R}$ & StA \\
\hline Dutch & Netherlands & Indo-European & $x$ & $\mathbf{x}$ & $(x)$ & Temp & $\mathrm{U}$ & 1 \\
\hline English & UK, USA, etc. & Indo-European & $x$ & $\mathbf{x}$ & $(x)$ & Temp & U & 1 \\
\hline Yimithirr & Australia & Pama Nyungan & & & $\mathbf{x}$ & TRF, TS & $\mathrm{R}$ & $H-G$ \\
\hline Hai//om & Namibia & Khoisan & $x$ & $(x)$ & $\mathbf{x}$ & $\mathrm{D}$ & $\mathrm{R}$ & $H-G$ \\
\hline Jaminjung & Australia & Jaminjungan & $x$ & $(x)$ & $(x)$ & $\mathrm{S}, \mathrm{T}$ & $\mathrm{R}$ & $H-G$ \\
\hline Japanese & Japan & Isolate & $x$ & $\mathbf{x}$ & $(x)$ & Temp & $U$ & I \\
\hline Kgalagadi & Botswana & Bantu & $\mathbf{x}$ & $x$ & $x$ & T St & $\mathrm{R}$ & StA \\
\hline Kilivila & Papua New Guinea & Austronesian & $\mathbf{x}$ & $\mathbf{x}$ & $\mathbf{x}$ & deN & $\mathrm{R}$ & ShA \\
\hline Longgu & Solomons & Austronesian & $x$ & $(x)$ & $x$ & TRF & $\mathrm{R}$ & ShA \\
\hline Tamil & India & Dravidian & $x$ & $\mathbf{x}$ & $x$ & $\mathrm{~S}$ & $U+R$ & StA \\
\hline Tiriyó & Brazil & Cariban & $\mathbf{x}$ & $\mathbf{x}$ & $\mathbf{x}$ & TRF & $\mathrm{R}$ & $\mathrm{H}, \mathrm{StA}$ \\
\hline Totonac & Mexico & Totonacan & $\mathbf{x}$ & & $(x)$ & Temp & $\mathrm{R}$ & ShA \\
\hline Tzeltal & Mexico & Mayan & $x$ & & $x$ & SubT, A & $\mathrm{R}$ & ShA \\
\hline Warwa & Australia & Nyulnyulan & $x$ & & $x$ & $\mathrm{D}$ & $\mathrm{R}$ & $\mathrm{H}-\mathrm{G}$ \\
\hline Yukatek & Mexico & Mayan & $x$ & $x$ & $x$ & TRF & $\mathrm{R}$ & ShA \\
\hline
\end{tabular}

Frame of reference: $x$ indicates that the corresponding Fo $R$ is used by a language. $(x)$ indicates that the FoR is only used in restricted circumstances, i.e. not in table-top space. $\mathbf{X}$ indicates the preferred FoR for describing spatial relationships between small-scale, manipulable objects (e.g. as in Figure 1). Ecological zone: $\mathrm{A}=$ alpine; $\mathrm{D}=$ desert; $\mathrm{deN}=$ denuded tropical rain forest; $\mathrm{H}=$ humid; $\mathrm{S}=$ savannah; SubT = subtropical; $\mathrm{St}=$ steppe; $\mathrm{T}=$ tropical; $\mathrm{TRF}=$ tropical rain forest; Temp = temperate. Dwelling: $\mathrm{R}=$ rural; $\mathrm{U}=$ urban; Subsistence mode: $\mathrm{H}=$ hunting; $\mathrm{H}-\mathrm{G}=$ hunter-gatherer; $\mathrm{ShA}=$ shifting agriculture; $\mathrm{StA}=$ stable agriculture; $\mathrm{I}=$ industrial. Data sources: Refs $[13,20]$ and Levinson, S.C. and Wilkins, D. Grammars of Space (unpublished).

language and cognition. This runs contrary to the assumption held by many cognitive scientists that spatial cognition is fundamentally egocentric $[3,4]$. But is the Relative FoR still somehow more 'natural'? For example, do children learn it more easily? The available evidence, although scant, does not support this hypothesis.

Children acquiring English, Italian, Serbo-Croatian and Turkish do not produce Relative uses of 'front/back' (e.g. 'the ball is in front of the tree') until around 5 years of age (Intrinsic uses appear a year or so earlier; e.g. 'in front of the man') [33]. The use of 'left' and 'right' to specify the location of one object with respect to another appears still later, at around 11 or 12 years [34].

Children learning an Absolute language acquire the relevant linguistic expressions just as early as children learning a Relative language - possibly even earlier. Tzeltal children produce Absolute vocabulary at two years, and by three-and-a-half they use Absolute vocabulary in an explicitly relational way; by 8 years they have fully acquired the Absolute linguistic system [35]. The early acquisition of Absolute vocabulary is also seen in studies of Tzotzil (Mexico) [36] and Balinese: 4-year-old Balinese children show Absolute behaviour not only in their language but also in non-linguistic tasks [20]. There is no evidence, then, for the hypothesis that the Relative FoR is privileged in child development.

\section{Conclusions}

Frames of reference - the most fundamental concepts underlying spatial cognition - seem unlikely things to 


\section{Box 2. Possible mechanisms of Whorfian effects}

Whorfian effects need be neither magical nor radical. There are several attested cognitive processes that could account for linguistic effects on non-linguistic representations, which in turn can have effects on the kind of computations carried out by the cognitive architecture $[38,39,43]$.

- Perceptual 'tuning' and attention. Experience, including experience with language, can influence the perceptual system such that it is more or less attuned to particular features in the environment $[44,45]$. For example, exposure to speech tunes the auditory system so that learners lose sensitivity to certain phoneme distinctions that are not present in their own language [46]. Similarly, although 9-month-old babies of different language backgrounds are sensitive to the distinction between tight-fit and loose-fit containment relations, by adulthood sensitivity depends on whether this distinction is relevant for the input language [47]. One attested mechanism through which language can produce such perceptual tuning is the selective direction of attention [48].

- Re-representation. Perceptual tuning can influence category formation early on in the learning process. But even when initial representations are robust and relatively consistent across learners, recoding can take place later. One well-known example is the recoding of representational units resulting from increased experience with a particular domain - the novice-expert shift $[49,50]$. The recoding of information into higher-order chunks circumvents the short-term memory bottleneck, and 'the most customary kind of recoding... is to translate into a verbal code' [51]. Across languages, perceptual features and dimensions are organized in many different ways, even in domains often assumed to be cognitively 'given', such as colour [52] and space [53]. In some cases these language differences might affect the initial

vary across languages and cultures. But recent crosslinguistic work establishes that they do. The work reviewed here also suggests that linguistic diversity aligns with cognitive diversity, as shown in people's languageindependent solutions to spatial tasks and unselfconscious gestures accompanying speech. Different frames of reference are acquired with comparable ease by children. Those sceptical of these findings often invoke ecological or cultural explanations; alternatively, they suggest that no known psychological mechanisms could account for such profound linguistic effects on cognition. We have argued that neither line of dismissal is plausible. We are left with findings that create problems for current models of the language-cognition interface (see Box 3 for some questions for future research). Rather than cognitive categories being universal and giving rise to universal semantic categories, as is typically supposed, it seems that cognitive categories are variable and they align with

\section{Box 3. Questions for future research}

- What are the neurocognitive underpinnings for linguistic frames of reference? How much plasticity is there?

- How do children learn linguistic frames of reference? And when do linguistic frames of reference begin to influence spatial cognition? - What are the cognitive consequences of being a bilingual in languages that rely on different frames of reference?

- Not all rural societies use an Absolute frame of reference, but urban languages appear to use a Relative frame of reference. Why is this?

- What mechanisms do speakers of Absolute languages use to keep track of directions in the Absolute frame of reference?

- Are speakers of Absolute languages better than speakers of Relative languages at view-independent object recognition? stages of concept formation (through 'perceptual tuning', above), whereas in others they might lead to 'representational redescription' over time [54].

- Structure-mapping. Another process that has been shown to affect the learning of categories is structure-mapping: the alignment of conceptual representations across situations, and the extraction of higher-order relational similarities [55]. Structure-mapping comes about through comparison of representations, and language can promote this comparison by drawing attention to 'what is to be compared to what' $[53,55]$. Use of the same word across different situations invites people to extract similarities, so that new categories are learnt. To the extent that languages classify situations according to different criteria, the kinds of similarities extracted, and consequently the categories acquired, will also differ [56-58].

- Costs of computation. Having different units of representation across languages, such as diverse semantic categories, can result in differences in the 'cost of computation' for otherwise equivalent input-output transformations [59]. When giving route directions, Japanese speakers sometimes point to geographically correct locations, suggesting they are using an Absolute FoR. But these pointing gestures are often preceded by glances to the visible part of the route and turns of the shoulder, which indicates that direction is in fact computed from visual memories of turns that are encoded in the Relative FoR [60]. Speakers of Absolute Languages produce gestures in the Absolute FoR unhesitatingly, without accompanying glances and shoulder shifts [13]. Thus, the same output might result from the use of different computations on different types of representations.

cross-linguistically variable semantic categories [28]. This work therefore contributes to the emerging view [37-39] that language can play a central role in the restructuring of human cognition.

\section{Acknowledgements}

This research was partly supported by European Union Marie Curie Fellowships, awarded to A.M. and D.B.M.H. The authors are solely responsible for information communicated and the European Commission is not responsible for any views or results expressed.

\section{References}

1 Kant, I. (1991) On the first ground of the distinction of regions in space. In The Philosophy of Right and Left (Van Cleve, J. and Frederick, R.E., eds), pp. 27-34, Kluwer

2 Miller, G.A. and Johnson-Laird, P.N. (1976) Language and Perception, Harvard University Press

3 Halligan, P.W. et al. (2003) Spatial cognition: evidence from visual neglect. Trends Cogn. Sci. 7, 125-133

4 Wang, R.F. and Spelke, E.S. (2002) Human spatial representation: insights from animals. Trends Cogn. Sci. 6, 376-382

5 Burgess, N. et al. (1999) Integrating hippocampal and parietal functions: a spatial point of view. In The Hippocampal and Parietal Foundations of Spatial Cognition (Burgess, N. and Jeffery, K.J., eds), pp. 3-32, Oxford University Press

6 Bickel, B. (1997) Spatial operations in deixis, cognition and culture: where to orient oneself in Belhare. In Language and Conceptualization (Nuyts, J. and Pederson, E., eds), pp. 46-83, Cambridge University Press

7 Danziger, E. (1996) Parts and their counterparts: spatial and social relationships in Mopan Maya. J. R. Anthropol. Inst. 2, 67-82

8 Mishra, R.C. et al. Ecology, language and performance on spatial cognitive tasks. Int. J. Psychol. 38 (in press)

9 Haviland, J.B. (1993) Anchoring, iconicity, and orientation in Guugu Yimithirr pointing gestures. J. Linguist. Anthropol. 3, 3-45

10 Haviland, J.B. (1998) Guugu Yimithirr cardinal directions. Ethos 26, 25-47

11 Levinson, S.C. (1996) Frames of reference and Molyneux's question: cross-linguistic evidence. In Language and Space (Bloom, P. et al., eds), pp. 109-169, MIT Press 
12 Levinson, S.C. (1997) From outer to inner space: linguistic categories and non-linguistic thinking. In Language and Conceptualization (Nuyts, J. and Pederson, E., eds), pp. 13-45, Cambridge University Press

13 Levinson, S.C. (2003) Space in Language and Cognition: Explorations in Cognitive Diversity, Cambridge University Press

14 Neumann, S. and Widlok, T. (1996) Rethinking some universals of spatial language using controlled comparison. In The Construal of Space in Language and Thought (Dirven, R. and Pütz, M., eds), pp. 345-372, de Gruyter

15 Pederson, E. (1995) Language as context, language as means: spatial cognition and habitual language use. Cognitive Linguistics 6, 33-62

16 Pederson, E. et al. (1998) Semantic typology and spatial conceptualization. Language 74, 557-589

17 Pederson, E. (2003) How many reference frames? In Spatial Cognition III: Routes and Navigation, Human Memory and Learning, Spatial Representation and Spatial Learning (Freska, C. et al., eds), pp. 287-304, Springer Verlag

18 Senft, G. (1997) Referring to Space: Studies in Austronesian and Papuan languages, Clarendon Press

19 Senft, G. (2001) Frames of reference in Kilivila. Studies in Language $25,521-555$

20 Wassmann, J. and Dasen, P.R. (1998) Balinese spatial orientation: some empirical evidence of moderate linguistic relativity. J. $R$. Anthropol. Inst. 4, 689-711

21 Widlok, T. (1997) Orientation in the wild: the shared cognition of the Hai//om Bushpeople. J. R. Anthropol. Inst. 3, 317-332

22 Levinson, S.C. (1994) Vision, shape and linguistic description: Tzeltal body-part terminology and object description. Linguistics 32, 791-855

23 van der Zee, E. and Slack, J. (2003) Representing Direction in Language and Space, Oxford University Press

24 Roberts, R.J. and Aman, C.J. (1993) Developmental differences in giving directions: spatial frames of reference and mental rotation. Child Dev. 64, 1258-1270

25 Gallistel, C.R. (2002) Language and spatial frames of reference in mind and brain. Trends Cogn. Sci. 6, 321-322

26 Gallistel, C.R. (2002) Conception, perception and the control of action. Reply to Majid. Trends Cogn. Sci. 6, 504

27 Levinson, S.C. et al. (2002) Returning the tables: language affects spatial reasoning. Cognition 84, 155-188

$28 \mathrm{Li}$, P. and Gleitman, L. (2002) Turning the tables: language and spatial reasoning. Cognition 83, 265-294

29 Bloom, P. and Keil, F.C. (2001) Thinking through language. Mind and language 16, 351-367

30 McNeill, D. (1992) Hand and Mind, Chicago University Press

31 Kita, S. and Ozyürek, A. (2003) What does cross-linguistic variation in semantic coordination of speech and gesture reveal? Evidence for an interface representation of spatial thinking and speaking. J. Mem. Lang. 48, 16-32

32 Kita, S. et al. (2001) Cultural specificity of spatial schemas as manifested in spontaneous gestures. In Spatial Schemas and Abstract Thought (Gattis, M., ed.), pp. 115-146, MIT Press

33 Johnston, J.R. (1988) Children's verbal representation of spatial location. In Spatial Cognition (Stiles-Davies, J. et al., eds), pp. 195206, Erlbaum

34 Piaget, J. (1928) Judgement and Reasoning in the Child, Routledge

35 Brown, P. and Levinson, S.C. (2000) Frames of spatial reference and their acquisition in Tenejapan Tzeltal. In Culture, Thought and Development (Nucci, L. et al., eds), pp. 167-197, Erlbaum

36 De León, L. (1994) Exploration in the acquisition of geocentric location by Tzotzil children. Linguistics 32, 857-884

37 Gumperz, J.J. and Levinson, S.C. (1996) Rethinking Linguistic Relativity, Cambridge University Press

38 Bowerman, M. and Levinson, S.C. eds (2001) Language Acquisition and Conceptual Development, Cambridge University Press

39 Gentner, D. and Goldin-Meadow, S. eds (2003) Language in Mind, MIT Press

40 Lindsey, D.T. and Brown, A.M. (2002) Color naming and the phototoxic effects of sunlight on the eye. Psychol. Sci. 13, 506-512

41 Brown, C.H. (1983) Where do cardinal direction terms come from? Anthropological Linguistics 25, 121-161

42 Greenfield, P.M. et al. (2003) Cultural pathways through universal development. Annu. Rev. Psychol. 54, 461-490

43 Lucy, J.A. Language, culture and mind. In Language, Culture and Mind (Achard, M. and Kemmer, S., eds), CSLI Publications (in press)

44 Goldstone, R.L. (1998) Perceptual learning. Annu. Rev. Psychol. 49, $585-612$

45 Sloutsky, V.M. (2003) The role of similarity in the development of categorization. Trends Cogn. Sci. 7, 246-251

46 Werker, J.F. and Tees, R.C. (1999) Influences on infant speech processing: toward a new synthesis. Annu. Rev. Psychol. 50, 509-535

47 McDonough, L. et al. (2003) Understanding spatial relations: flexible infants, lexical adults. Cogn. Psychol. 46, 229-259

48 Smith, L.B. et al. (2002) Object name learning provides on-the-job training for attention. Psychol. Sci. 13, 13-19

49 Bailenson, J.N. et al. (2002) A bird's eye view: biological categorization and reasoning within and across cultures. Cognition 84, 1-53

50 Ross, N. et al. (2003) Cultural and experiential differences in the development of folkbiological induction. Cogn. Dev. 18, 25-47

51 Miller, G.A. (1956) The magical number 7, plus or minus 2: some limits on our capacity for processing information. Psychol. Rev. 63, 81-97

52 Davidoff, J. (2001) Language and perceptual categorisation. Trends Cogn. Sci. 5, 382-387

53 Bowerman, M. and Choi, S. (2003) Space under construction language-specific spatial categorization in first language acquisition. In Language in Mind (Gentner, D. and Goldin-Meadow, S., eds), pp. 387-427, MIT Press

54 Karmiloff-Smith, A. (1992) Beyond Modularity: a Developmental Perspective on Cognitive Science, MIT Press

55 Gentner, D. (2003) Why we're so smart. In Language in Mind (Gentner D. and Goldin-Meadow, S., eds), pp. 195-235, MIT Press

56 Lucy, J.A. and Gaskins, S. (2001) Grammatical categories and the development of classification preferences: a comparative approach. In Language Acquisition and Conceptual Development (Bowerman, M. and Levinson, S.C., eds), pp. 257-283, Cambridge University Press

57 Boroditsky, L. et al. (2003) Sex, syntax and semantics. In Language in Mind (Gentner, D. and Goldin-Meadow, S., eds), pp. 61-79, MIT Press

58 Boroditsky, L. (2001) Does language shape thought? Mandarin and English speakers' conceptions of time. Cogn. Psychol. 43, 1-22

59 Hunt, E. and Agnoli, F. (1991) The Whorfian hypothesis: a cognitive psychology perspective. Psychol. Rev. 98, 377-389

$60 \mathrm{Kita}$, S. (2003) Interplay of gaze, hand, torso orientation, and language in pointing. In Pointing: Where Language, Culture, and Cognition Meet (Kita, S., ed.), pp. 293-306, Erlbaum

\section{Articles of interest in Trends in Neurosciences}

- An action video game modifies visual processing, by Maximilian Riesenhuber Trends in Neurosciences 27, 72-74

- Psychology and neurobiology of simple decisions, by Philip L. Smith and Roger Ratcliff

Trends in Neurosciences 27 doi:10.1016/j.tins.2004.01.006

- The aperture problem in egocentric motion, by Ikuya Murakami Trends in Neurosciences 27 doi:10.1016/j.tins.2004.01.009 